\title{
Impact of prehospital rapid sequence intubation and mechanical ventilation on prehospital vital signs and outcome in trauma patients
}

\author{
1 Center for Emergency Medicine Maribor, Maribor, Slovenia \\ 2 Medical Faculty, University of Maribor, Maribor, Slovenia \\ Correspondence to: \\ Tine Pelcl \\ Prehospital Unit \\ Center for Emergency Medicine \\ Ulica talcev 9, 2000 Maribor, Slovenia \\ Phone: (+386) 41576250 , \\ E-mail: tpelcl1@gmail.com
}

TINE PELCL ${ }^{1}$, VESNA BOROVNIK LESJAK ${ }^{1}$, VITKA VUJANOVIĆ ${ }^{1}$, MATEJ STRNAD ${ }^{2}$

\section{ABSTRACT}

Introduction. Medications during rapid sequence intubation (RSI) have known detrimental side effects. Prehospital mechanical ventilation after successful endotracheal intubation also increases mortality due to hyperventilation and positive pressure ventilation. The aim of this retrospective analysis was to determine the impact of RSI on prehospital hemodynamic parameters and prehospital ventilation status on mortality rate and functional outcome in trauma patients.

Methods. Charts of 73 trauma patients, who underwent prehospital RSI over a 12year period, were retrospectively reviewed. Prehospital vital signs, before and after RSI, were compared. Patients were divided, according to ventilation status, into three groups based on initial $\mathrm{PaCO}$ 2: hypocarbic/hyperventilated $(\mathrm{PaCO} 2<35 \mathrm{mmHg})$, normocarbic/normoventilated $\quad(\mathrm{PaCO} 2$ $35-45 \mathrm{mmHg}$ ) and hypercarbic/hypoventilated (PaCO2 $>45 \mathrm{mmHg}$ ).

Results. Seventy-three patients were enrolled in the retrospective analysis. There was a significant difference in respiratory rate $(\mathrm{p}=0.046)$, arterial oxygen saturation $(\mathrm{p}<0.001)$, mean arterial pressure $(\mathrm{p}<0.001)$ and Glasgow Coma Scale (GCS) $(\mathrm{p}<0.001)$ before and after RSI. GCS at discharge $(p=0.003)$ and arterial oxygen saturation $(\mathrm{p}=0.05)$ were significantly higher in the normoventilated group. There was no significant difference in survival to hospital discharge among compared groups.

Conclusion. Our retrospective analysis suggests that prehospital RSI has no detrimental hemodynamic side effects and that normoventilation leads to a favorable neurological outcome.
Key words: intubation, prehospital, mechanical ventilation, trauma, hemodynamics

\section{INTRODUCTION}

Endotracheal intubation (ETI) using the rapid sequence intubation (RSI) protocol was introduced to prehospital Emergency Medical Services (EMS) to secure the airway, thus preventing aspiration. Aggressive early airway management, using medications during the RSI protocol, increases the success of prehospital ETI. $(1,2)$ Drugs used for RSI have known detrimental side effects, such as hypotension (3), which could worsen the outcome in trauma patients. (4) Some drugs used for RSI have a greater effect on hemodynamics than others. It has been shown that thiopental causes a greater fall in blood pressure than midazolam or etomidate, thereby reducing cerebral perfusion pressure and contributing to secondary brain injury. Fentanyl has the most neutral hemodynamic profile. (5) On the other hand, ETI and prehospital ventilation, surprisingly, also increase mortality rate. $(6,7)$ Suggested causes for this finding are hyperventilation (8) and positive pressure ventilation, (9) that both have detrimental effects on hemodynamic parameters resulting in hypotension and worsening ischemia.

One of the goals of prehospital ventilation with pure oxygen after successful ETI is to prevent hypoxia, which is one of the wellknown secondary insults. Its detrimental effects, especially in patients with severe traumatic brain injury, are well established. (10) Deleterious effects of hypoxia were recognized by guidelines and it was recommended to avoid hypoxia and thus secondary brain injury. (11)

The purpose of this single center retrospective analysis was to determine a) the impact of RSI on prehospital hemodynamic parameters and b) the impact of prehospital ventilation status on mortality rate and functional outcome at discharge from hospital in trauma patients in an emergency physician-led prehospital EMS.

\section{MATERIALS AND METHODS}

\section{Statement of human rights}

The retrospective analysis was approved by the National Medical Ethics Committee of the Republic of Slovenia, which decided that the analysis could be conducted without informed consent from participants.

\section{Study design}

Charts of trauma patients, who underwent ETI using the RSI method between January 2000 and December 2012, were retrospectively reviewed. Authors would like to emphasize that our emergency physicians working in EMS are well trained in advanced life support skills and have been using the RSI protocol to secure airways since the beginning of 2000. Since then, all trauma patients who cannot adequately oxygenate pulmonary capillary blood or with a compromised airway or ventilation and Glasgow Coma Scale (GCS) less than 8 at the scene, undergo prehospital ETI using the RSI method. Endotracheal placement of the tube is confirmed by measuring the partial pressure of end tidal carbon dioxide 
Table 1. Baseline characteristics.

\begin{tabular}{ll}
\hline Male sex (\%) & 77 \\
\hline Age (years) & $33,4(20,5)$ \\
\hline ISS & $33,37(19,65)$ \\
\hline Fluids used during transport to ED: & $614(233)$ \\
\hline $\mathbf{0 , 9} \% \mathbf{~ N a C l ~ s o l u t i o n ~ ( m l ) ~}$ & $620(288)$ \\
\hline HAES (ml) & $250(0)$ \\
\hline HyperHAES (ml) & $21,9(5,5)[61]$ \\
\hline Drugs used for RSI: & $466,7(57,7)[3]$ \\
\hline Etomidate (mg) & $6,7(4,3)[58]$ \\
\hline Thiopental (mg) & $0,121(0,066)[34]$ \\
\hline Midazolam (mg) & $93,8(16,5)[58]$ \\
\hline Fentanyl (mg) & $7,1(1,9)[53]$ \\
\hline Succinylcholine (mg) & $11,1(3,1)[9]$ \\
\hline Vecuronium (mg) & $59,6(52,1)[6]$ \\
\hline Piritramide (mg) & \\
\hline Ketamine (mg) &
\end{tabular}

Data are presented as mean (SD). Numbers in square brackets denote the number of patients receiving the drug.

ED, Emergency Department; HAES, hydroxyethyl starch; HyperHAES, 7,2\% NaCl/6\% hydroxyethyl starch; ISS, Injury Severity Score.

Table 2. Comparison of prehospital vital signs before and after RSI

\begin{tabular}{lll}
\hline & Before RSI & After RSI \\
\hline $\begin{array}{l}\text { Arterial Oxygen Saturation } \\
(\%)\end{array}$ & $84(13)$ & $97(5)$ \\
\hline $\begin{array}{l}\text { Mean Arterial Pressure } \\
(\mathrm{mmHg})\end{array}$ & $90(24)$ & $99(22)$ \\
\hline Respiratory Rate (min-1) & $12(6)$ & $11(1,6)$ \\
\hline Heart Rate (min-1) & $92(21)$ & $84(17)$ \\
\hline Glasgow Coma Scale Score & $6(3)$ & $3(0)$ \\
\hline
\end{tabular}

Data are presented as mean (SD). RSI, Rapid sequence intubation.

(petCO2). Patients who undergo prehospital ETI are mechanically ventilated using transport ventilator Weinmann ${ }^{\star}$ Medumat with intermittent positive-pressure ventilation (IPPV) mode and continuous end tidal CO2 (etCO2) monitoring. Parameters (minute volume, respiratory frequency) on transport ventilators with inspired oxygen concentration of $100 \%$ are selected by emergency physicians. All patients enrolled in the retrospective analysis were successfully intubated in the first attempt. There was no misplaced intubation. Trauma patients were treated according to the current trauma life support guidelines and were transported directly to the Emergency Department (ED) and then admitted to the surgical Intensive Care Unit (ICU) of the University Clinical Center of Maribor. Arterial blood gas analysis was performed in the hospital central laboratory on ad- mission to the ED. Arterial carbon dioxide partial pressure $(\mathrm{PaCO} 2)$ values were obtained from initial arterial blood gas analysis immediately on arrival to the ED. The population was sorted into three groups according to ventilation status, based on initial PaCO2: hypocarbic/hyperventilated $(\mathrm{PaCO} 2<35 \mathrm{mmHg})$, normocarbic/normoventilated (PaCO2 $35-45 \mathrm{mmHg}$ ) and hypercarbic/hypoventilated $(\mathrm{PaCO} 2>45$ $\mathrm{mmHg}$ ). Six patients were excluded from the analysis according to ventilation status due to missing data (no arterial blood gas analysis on admission). Mortality rate was defined by in-hospital death of any cause. Functional outcome was measured using Glasgow Outcome Scale (GOS), Glasgow Pittsburgh Cerebral Performance Categories Scale (CPC) and GCS level at discharge from hospital.

Prehospital vital signs (Glasgow Coma
Scale, heart rate, respiratory rate, oxygen saturation, mean arterial pressure) were recorded before and after RSI.

\section{STATISTICAL ANALYSIS}

SigmaPlot 11.0 (Systat Software, Point Richmond, CA) was used for the statistical analysis. Paired t-test was used to compare parameters before and after RSI. One-way ANOVA was used to compare differences among the three groups according to ventilation status. The data were presented as means (SD). A two-tailed value of $\mathrm{p}<0.05$ was considered significant.

\section{RESULTS}

Baseline characteristics are presented in table 1 . Seventy-three patients were enrolled in the retrospective analysis. Forty patients sustained multiple trauma while 33 patients suffered isolated head injury.

When comparing parameters before and after RSI for all patients included in the retrospective analysis (table 2), there were significant differences in respiratory rate $(\mathrm{p}=0.046)$, arterial oxygen saturation $(\mathrm{p}<0.001)$, mean arterial pressure $(\mathrm{p}<0.001)$ and Glasgow Coma Scale $(p<0.001)$. The decrease in heart rate after RSI was not significant ( $\mathrm{p}=0.096)$.

Comparison of parameters according to ventilation status among the three groups is presented in Table 3. GCS at discharge was significantly higher in the normoventilated/normocarbic group compared to the hyperventilated/hypercarbic group $(\mathrm{p}=0.003)$. Arterial oxygen saturation was also significantly different among the three groups ( $p=0.05$ ), while the difference in CPC was defined as statistically insignificant by One-way ANOVA test despite a numerically identical $\mathrm{p}$-value $(\mathrm{p}=0.05)$. There were no statistically significant differences in other observed parameters.

\section{DISCUSSION}

This retrospective analysis investigates the impact of ETI using the RSI protocol on hemodynamic parameters in the prehospital environment in trauma patients. Our results show that mean arterial oxygen saturation and blood pressure significantly increase after ETI. These findings suggest that ETI and prehospital ventilation are successful procedures in the prehospital arena for avoiding well known secondary insults, like hypoxia and hypotension. Epi- 
Table 3. Comparison of parameters according to ventilation status

\begin{tabular}{|c|c|c|c|c|}
\hline & $\begin{array}{l}\text { Hyperventilated/ } \\
\text { Hypocarbic }\end{array}$ & $\begin{array}{l}\text { Normoventilated/ } \\
\text { Normocarbic }\end{array}$ & $\begin{array}{l}\text { Hypoventilated/ } \\
\text { Hypercarbic }\end{array}$ & P-value \\
\hline Sample size & 12 & 37 & 18 & \\
\hline Age (years) & $53(22)$ & $42(20)$ & $44(20)$ & 0,257 \\
\hline Heart rate $(\min -1)$ & $72(21)$ & $87(15)$ & $84(11)$ & 0,275 \\
\hline $\begin{array}{l}\text { Respiratory rate } \\
(\mathrm{min}-1)\end{array}$ & $11(2)$ & $12(2)$ & $11(1)$ & 0,419 \\
\hline MAP (mmHg) & $87(25)$ & $93(21)$ & $87(29)$ & 0,718 \\
\hline $\mathrm{SpO} 2(\%)$ & $99(1)$ & $98(4)$ & $95(7)$ & 0,05 \\
\hline ISS & $43(25)$ & $31(20)$ & $32(16)$ & 0,19 \\
\hline $\begin{array}{l}\text { AISH > } 3 \text { (1-yes, } \\
2 \text {-no) }\end{array}$ & $1(0)$ & $1(0)$ & $1(0)$ & 0,733 \\
\hline $\begin{array}{l}\text { STHD (1-yes, } \\
2 \text {-no) }\end{array}$ & $1(1)$ & $1(0)$ & $2(1)$ & 0,087 \\
\hline GOS & $3(2)$ & $3(2)$ & $2(2)$ & 0,094 \\
\hline GCS at discharge & $12(4)$ & $15(1)$ & $14(2)$ & 0,003 \\
\hline $\mathrm{CPC}$ at discharge & $2(1)$ & $1(1)$ & $2(1)$ & 0,05 \\
\hline
\end{tabular}

Data are presented as mean values (SD). AISH, Abbreviated Injury Scale for Head; CPC, Cerebral Performance Category Scale; GCS, Glasgow Coma Score; GOS, Glasgow Outcome Score; ISS, Injury Severity Score; MAP, mean arterial pressure; SpO2, arterial oxygen saturation; STHD, Survival to the hospital discharge.

sodes of hypoxia are a common secondary insult in the prehospital setting and ICU and increase the mortality rate and degree of disability at discharge. $(12,13)$ Duration of the hypoxic episode also plays a role. Longer hypoxic episodes increase the mortality rate. $(12,14)$ Studies have also shown that there are many patients' predisposing factors that cause hypotension after RSI (3) and that peri-intubation hypotension significantly increases in-hospital mortality. (15) Our data also suggest that drugs used in our retrospective analysis for RSI (in most cases a combination of sedatives, etomidate, as an induction agent, and midazolam, to maintain sedation, together with muscle relaxants, succinylcholine and vecuronium, to maintain prolonged muscle relaxation) and their possible side effects affecting hemodynamics play a minor role. Even more, an increase in mean arterial pressure after RSI was found. Inappropriate sedation and pain as possible causes of raised mean arterial pressure could be excluded. Authors assume that patients from our retrospective analysis who underwent RSI were properly sedated, demonstrated by a significant decrease in GCS level (all patients had GCS level 3 after sedation) and a significant decrease in heart rate. It was shown that heart rate variability correlates with level of analgesia. $(16,17)$ Medications used for RSI in our retrospective analysis along with prehos- pital fluid resuscitation did not show the possible detrimental hemodynamic side effects that could compromise a favorable outcome. (18) Data from our retrospective analysis are supported by other studies which showed that etomidate preserves hemodynamic status compared to other sedatives. $(5,19)$

The possible beneficial effect of early outof-hospital intubation to secure the airway and to prevent hypoxia and aspiration has been questioned. Increased mortality was observed when prehospital intubation was performed by paramedics with limited ETI training and clinical skills, and with limited access to RSI drugs. $(6,7,20,21)$ Furthermore, even patients with traumatic brain injury who underwent paramedic RSI had increased mortality, due to hypoxia and hyperventilation during RSI. (22) On the other hand, studies showed that ETI performed in the ED by emergency physicians $(7,20)$ or by air ambulance personnel using neuromuscular blocking agents had favorable outcomes, decreasing mortality. $(23,24)$ No difference was observed in patient outcome after prehospital ETI by aeromedical crew using the RSI protocol compared to undergoing ETI in a trauma resuscitation suite. (25) It is reasonable to assume that the procedure (ETI) itself and possible complications $(22,26,27)$ during ETI are connected with patient outcome. Deleterious effects of prehospital intuba- tion and ventilation are associated with the risk of hyperventilation and positive pressure ventilation. Hypocapnia due to hyperventilation causes cerebral vasoconstriction of precapillary resistance vessels and can result in global ischemia through a decrease in cerebral blood flow and diminished brain oxygenation which could result in a less favorable neurological outcome in these patients. Positive pressure ventilation increases intrathoracic pressure, which reduces venous return and decreases blood pressure and cardiac output. $(9,22,28)$ However, hyperventilation reduces intracranial pressure through vasoconstriction (29) and is a recommended treatment in patients with acute brainstem herniation. (30)

The proposed mechanism for the detrimental effect of hypoventilated/hypercarbic patients is suboptimal vasoreactivity to carbon dioxide (CO2), with preserved $\mathrm{CO} 2$ vasoreactivity to hyperventilation, but buffered vasodilatation to hypoventilation. This would cause relative ischemia precipitating secondary brain injury in both hypocarbic and hypercarbic patients. (31)

Data from our retrospective analysis suggest that ventilation status has a significant impact on arterial oxygen saturation and neurological functional outcome in trauma patients. These findings show that normoventilation in trauma patients' preserves favorable hemodynamic status and thus better outcome and are in accordance with above mentioned studies. A high percentage ( $45 \%$ ) of patients in our retrospective analysis was not optimally ventilated and that fact raises some questions. This could be due to over- or under estimation of patient's ideal body weight and high frequency ventilation consequently leading to inappropriate prehospital ventilation. Use of blood gas measurements of $\mathrm{pCO} 2$ with portable devices in the prehospital environment, which are acceptable in guiding protocol-directed ventilator management, should be considered. (32)

Our retrospective analysis has several limitations. Firstly, this is a non-randomized retrospective analysis. The nature of the retrospective analysis by itself could include some biases. In the present study analysis of the data collected from January 2000 to December 2012 was done. Progress over the years in in-hospital care of severely injured patients may affect the rate of in-hospital mortality. However, ambulance treatment protocols remained mostly the same throughout the analysis period. Secondly, the sample size is small. Thirdly, 
arterial blood gas analysis (pCO2) at arrival in the ED was used as a surrogate for ventilation status.

\section{CONCLUSIONS}

Our retrospective analysis suggests that prehospital RSI in emergency physicianled EMS, together with prehospital fluid resuscitation, has no detrimental hemodynamic side effects, potentially resulting in hypotension and thereby worsening functional outcome in trauma patients. Oxygen saturation also improved after prehospital RSI.

Normoventilation/normocarbia leads to a favorable neurological outcome and should be maintained by ventilator set- tings and controlled with measurements of $\mathrm{pCO} 2$ in the prehospital environment, using a portable device.

However, our data should be interpreted with caution due to the small sample size. Larger prospective studies are necessary to validate these findings.

\section{REFERENCES}

1. Mace SE. Challenges and advances in intubation: rapid sequence intubation. Emerg Med Clin North Am 2008;26:1043-68.

2. Ballow SL, Kaups KL, Anderson S, Chang M. A standardized rapid sequence intubation protocol facilitates airway management in critically injured patients. J Trauma Acute Care Surg 2012;73:1401-5.

3. Lin CC, Chen KF, Shih CP, Seak CJ, Hsu KH. The prognostic factors of hypotension after rapid sequence intubation. Am J Emerg Med 2008;26:845-51.

4. Shapiro NI, Kociszewski C, Harrrison T, Chang Y, Wedel SK, Thomas SH. Isolated prehospital hypotension after traumatic injury: a predictor of mortality? J Emerg Med 2003;25:175-9.

5. Sivilotti MLA, Ducharme J. Randomized, double-blind study on sedatives and hemodynamics during rapid-sequence intubation in the emergency department: The SHRED Study. Ann Emerg Med 1998:313-24.

6. Murray JA, Demetriades D, Berne TV, Stratton SJ, Cryer HG, Bongard F, et al. Prehospital intubation in patients with severe head injury. J Trauma 2000; 49:1065-70.

7. Davis DP, Peay J, Sise MJ, Vilke GM, Kennedy F, Eastman AB, et al. The impact of prehospital intubation on outcome in moderate to severe traumatic brain injury. J Trauma 2005; 58:933-9.

8. Muizelaar JP, Marmarou A, Ward JD, Kontos HA, Choi SC, Becker DP, et al. Adverse effects of prolonged hyperventilation in patients with severe head injury: a randomized clinical trial. J Neurosurg 1991; 75:731-9.

9. Pepe PE, Raedler C, Lurie KG, Wiggintoon JG. Emergency ventilator management in severe hemorrhagic states: elemental or detrimental? J Trauma 2003; 54:1048-55.

10. Chesnut RM, Marshall LF, Klauber MR, Blunt BA, Baldwin N, Eisenberg HM, et al. The role of secondary brain injury in determining outcome from severe head injury. J Trauma 1993; 34:216-22.

11. The Brain Trauma Foundation, American Association of Neurological Surgeons, Congress of Neurological Surgeons. 2007; Joint Section on Neurotrauma and Critical Care; AANS/CNS. Guidelines for the management of severe traumatic brain injury. VI. Indications for intracranial pressure monitoring. J Neurotrauma 2007;24:S37-S44.

12. Chi JH, Knudson MM, Vassar MJ, McCarthy MC, Shapiro MB, Mallet S, et al. Prehospital hypoxia affects outcome in patients with traumatic brain injury: A prospective multicenter study. J Trauma 2006; 61:1134-41.

13. Chang JJ, Youn TS, Benson D, Mattick H, Andrade N, Harper CR, et al. Physiologic and functional outcome correlates of brain tissue hypoxia in traumatic brain injury. Crit Care Med 2009;37:283-90.

14. Valadka AB, Gopinath SP, Contant CF, Uzura M, Robertson CS. Relationship of brain tissue PO2 to outcome after severe head injury. Crit Care Med 1998; 26:1576-81.

15. Heffner AC, Swords DS, Nussbaum ML, Kline JA, Jones AE. Predictors of the complication of postintubation hypotension during emergency airway management. J Crit Care 2012;27:587-93.

16. Jeanne M, Logier R, De Jonckheere J, Tavernier B. Validation of a graphic measurement of heart rate variability to asses analgesia/ nociception balance during general anesthesia. Conf Proc IEEE Eng Med Biol Soc 2009;2009:1840-3.

17. Logier R, Jeanne M, Tavernier B, De Jonckheere J. Pain/analgesia evaluation using heart rate variability analysis. Conf Proc IEEE Eng Med Biol Soc 2006;1:4303-6.

18. Kogler VM, Deutsch J, Sakan S. Analgesia and sedation in hemodynamic unstable patient. Signa Vitae 2008;3:S10-S12.

19. Choi YF, Wong TW, Lau CC. Midazolam is more likely to cause hypotension then etomidate in emergency department rapid sequence intubation. Emerg Med J 2004 21:700-2.

20. Wang HE, Peitzman AB, Cassidy LD, Adelson PA, Yealy DM. Out-of-hospital endotracheal intubation and outcome after traumatic brain injury. Ann Emerg Med 2004;44:439-50.

21. Eckstein M, Chan L, Schneir A, Palmer R. Effect of prehospital advanced life support on outcomes of major trauma patients. J Trauma 2000;48:643-8.

22. Davis DP, Dunford JV, Poste JC, Ochs M, Holbrook T, Fortlage D, et al. The impact of hypoxia and hyperventilation on outcome after paramedic rapid sequence intubation of severely head-injured patients. J Trauma 2004;57:1-8.

23. Wang HE, Balasubramani GK, Cook LJ, Lave JR, Yealy DM. Out-of-hospital endotracheal intubation experience and patients outcomes. Ann Emerg Med 2010; 55:527-37.

24. Davis DP, Peay J, Sise MJ, Kennedy F, Simon F, Tominaga G, et al. Prehospital airway and ventilation management: A trauma score and injury severity score-based analysis. J Trauma 2010;69:294-301.

25. Sloane C, Vilke GM, Chan TC, Hayden SR, Hoyt DB, Rosen P. Rapid sequence intubation in the field versus hospital in trauma patients. J Emerg Med 2000;19:259-64.

26. Katz SH, Falk JL. Misplaced endotracheal tubes by paramedics in an urban emergency medical services system. Ann Emerg Med 
2001;37:32-7.

27. Dunford JV, Davis DP, Ochs M, Doney M, Hoyt DB. Incidence of transient hypoxia and pulse rate reactivity during paramedic rapid sequence intubation. Ann Emerg Med 2003;42:721-8.

28. Davis DA, Idris AH, Sise MJ, Kennedy F, Eastman AB, Velky T, et al. Early ventilation and outcome in patients with moderate to severe traumatic brain injury. Crit Care Med 2006;34:1202-8.

29. Rangel-Castilla L, Rivera Lara L, Gopinath S, Swank PR, Valadka A, Robertson C. Cerebral hemodynamic effects of acute hyperoxia and hyperventilation after severe traumatic brain injury. J Neurotrauma 2010;27:1853-63.

30. The Brain Trauma Foundation, American Association of Neurological Surgeons, Congress of Neurological Surgeons. 2007; Joint Section on Neurotrauma and Critical Care; AANS/CNS. Guidelines for the management of severe traumatic brain injury. XIV. Hyperventilation. J Neurotrauma 2007;24:S87-S90.

31. Dumont TM, Visioni AJ, Rughani AI, Tranmer BI, Crookes B. Inappropriate prehospital ventilation in severe traumatic brain injury increases in hospital mortality. J Neurotrauma 2010;27:1233-41.

32. Thomas FO, Hoffman TL, Handrahan DL, Crapo RO, Snow G. The measure of treatment agreement between portable and laboratory blood gas measurements in guiding protocol-driven ventilator management. J Trauma 2009;67:303-13. 